\title{
Characterization of the Sorption and Desorption of Lindane to Chitin in Seawater Using Reversible and Resistant Components
}

\author{
J. Magdalena Santana-Casiano and Melchor Gonzälez-Dảvila* \\ Universidad de Las Palmas de Gran Canaria, Facultad de Ciencias del Mar. Departamento de Quimica, \\ Las Palmas G.C.. P.O. Box 550, Spain
}

\begin{abstract}
- The lindane sorption-desorption experiments conducted using chitin as an adsorbent in seawater show that the partition coefficients decrease as adsorbent concentration increases and that sorption and desorption are not completely reversible at chitin concentrations lower than 10 $\mathrm{g} / \mathrm{L}$. These two effects have been analyzed in terms of a reversible-resistant two-component model of sorptiondesorption. Further studies varying the temperature (from 5 to $45^{\circ} \mathrm{C}$ ) and salinity (from 15 to $36 \%$ ) were carried out to clarify the mechanisms responsible for these effects. The partition coefficients for the resistant and reversible components are found to behave quite regularly in all the experimental conditions: the reversible component is fairly constant in the range of $45 \pm 4 \mathrm{~L} / \mathrm{kg}$ whereas the resistant component partition coefficient is inverse in relation to the chitin concentration, and it approaches zero when the chitin concentration is held at values higher than $10 \mathrm{~g} / \mathrm{L}$. The temperature and salinity do not affect the reversible component partition coefficient, and it is shown that the resistant component decreases both as temperature increases and as salinity decreases. Lindane sorption in different types of sites and to differing degrees can account for the observed effects.
\end{abstract}

\section{Introduction}

The interaction between organic pollutants and soils and other natural adsorbents in an aqueous environment is a mechanism of fundamental importance in determining the fate and distribution of these materials in the environment. Many sorption-desorption experiments have been performed and designed in order to characterize this interaction. However, it is important to note that only some studies have been found in the literature concerning seawater-particle interactions. Most of the studies used natural waters and distilled water and only a few of them took into account ionic strength effects. The equilibrium condition defines the ratio between the solid-phase and dissolved concentrations, which is referred to as the partition coefficient. In studies conducted to measure partition coefficients and characterize sorption-desorption behavior, several apparent anomalies have been detected, which have not, as yet, been adequately explained. Numerous sorption studies (e.g., Grover and Hance (1), O'Connor and Connolly (2), Di Toro et al. (3), Voice et al. (4), Bowman and Sans (5), and Coates and Elzerman (6)) have demonstrated that the equilibrium partition coefficient decreases as the ratio of solids to water increases. A variety of results on organic chemical-soil systems have suggested that sorption is not a totally reversible process. In these cases, the system shows sorption-desorption hysteresis or nonsingularity (7-11). Rao and Davidson (7) suggested that the hysteresis effects were due to experimental errors. Di Toro et al. (12) modified sorption and desorption experiments to test these experimental factors and concluded that they were not the cause of hysteresis. Di Toro and Horzempa (8) modeled sorption-desorption of hexachlorobiphenyl (HCB) on lake sediments and montmorillonite clay by defining reversible and resistant components for the sorbed state. Gschwend and Wu (13) and Baker et al. (14) have pointed out that nonsettling organic material and dissolved macromolecules can fully explain the solid concentration effect and the nonreversible sorption phenomena observed. All of this suggests that equilibrium environmental speciation of hydrophobic organic compounds (HOC) should include three "phases": dissolved, sorbed to nonsettling particles or macromolecules, and sorbed to settling solids. However, Jaffe (15) and Witkowski et al. (16) have demonstrated for malathion and Aroclor 1242, respectively, that the hysteresis effect is not exclusively attributable to the presence of nonsettling particles. Chin et al. (17) and Caron et al. (18) have shown that the presence of dissolved or suspended organic macromolecules in natural waters could explain the solid effect but only for very hydrophobic organic substances with $K_{\mathrm{ow}}>10^{5}$. The sorption of HOC with $K_{\mathrm{ow}}$ less than $10^{5}$ is relatively insensitive to the type and environmentally observed concentration of organic polymers dispersed in the aqueous phase.

In this work, we show the results obtained for lindane sorption-desorption to chitin in seawater. Lindane $(\gamma$ $1,2,3,4,5,6$-hexachlorocyclohexane) is a lightly polar, chlorinated insecticide with low volatility (Henry's constant, $\left.K_{\mathrm{H}}=4.8 \times 10^{-7} \mathrm{~m}^{3} \mathrm{~atm}^{-1} \mathrm{~mol}\right)$ and moderate hydrophobicity $\left(\log K_{\text {ow }}=3.72\right)(19)$. Chitin is the most common polysaccharide in nature whose basic unit is an amino sugar. Chitin is insoluble in water, organic solvents, concentrated alkali, or dilute mineral acids, but it can be solubilized in concentrated mineral acids. It is one of the constituents of the exoskeleton of most of the invertebrates (20), and it is consequently abundant in the natural environment. Chitin properties and uses can be found elsewhere $(21-23)$. The experiments focused on the two phenomena: the reversibility of sorption and desorption, and the effect of adsorbent mass on the extent of partitioning, taking into account the effect of nonsettling particles. According to Di Toro and Horzempa (8), the process has been consistently described as if the sorbed lindane were assumed to be the composition of two components: a reversible component which readily sorbs and desorbs reversibly, and a resistant component which resists desorption during the first consecutive desorption and may or may not ultimately desorb during consecutive desorption. The effect of temperature and salinity were also investigated to give us a greater insight into these phenomena.

\section{Experimental Section}

The chitin powder used was from crab shells and obtained from Sigma. Carbon content by using the Walkley-Black procedure (24) was found to be $40.0 \%$, a value close to those given for humic acids. It made chitin a useful organic-phase model for predicting sorption-desorption processes in a marine environment. Chitin acid-base properties have been described prior to this work (21). In some cases, the chitin used for equilibrations was prewashed to remove nonsettling microparticles and soluble macromolecules. This washing was done by suspending the chitin in MilliQ water (MilliQ system, Millipore, Inc), shaking for $24 \mathrm{~h}$, and discarding the supernatant. After 
Table I. Sorption and Desorption Partition Coefficients and the Coefficients for the Reversible-Resistant Two-Component Model for the Sorption of Lindane to Chitin in Seawater ${ }^{4}$

\begin{tabular}{|c|c|c|c|c|c|c|}
\hline \multirow[b]{2}{*}{ [chitin], g/L } & \multicolumn{6}{|c|}{ coefficients, L/ $\mathrm{kg}$} \\
\hline & K, & $K_{\mathrm{d}}$ & $K_{\text {s }}$ & $K_{s}$ & $K_{\circ}{ }^{b}$ & $K_{x}^{b}$ \\
\hline 2.5 & $80.08 \pm 4$ & $430.2 \pm 18$ & $38.73 \pm 2$ & $43.39 \pm 2$ & $37.48 \pm 4$ & $42.7 \pm 3$ \\
\hline 4 & $67.33 \pm 4$ & $234.0 \pm 28$ & $27.84 \pm 3$ & $41.02 \pm 3$ & $26.93 \pm 5$ & $40.4 \pm 4$ \\
\hline 6.25 & $62.07 \pm 3$ & $106.1 \pm 4$ & $17.97 \pm 2$ & $47.06 \pm 1$ & $15.98 \pm 2$ & $48.7 \pm 3$ \\
\hline $6.25(1)^{c}$ & $42.09 \pm 2$ & $42.8 \pm 7$ & $0.11 \pm 1$ & $44.45 \pm 1$ & $0.18 \pm 3$ & $41.0 \pm 5$ \\
\hline $6.25(2)^{c}$ & $78.68 \pm 7$ & $186.3 \pm 30$ & $31.97 \pm 8$ & $46.43 \pm 1$ & $31.64 \pm 2$ & $47.1 \pm 2$ \\
\hline $6.25(1)^{d}$ & $49.01 \pm 2$ & $52.1 \pm 4$ & $0.86 \pm 1$ & $49.80 \pm 1$ & $0.91 \pm 1$ & $48.0 \pm 2$ \\
\hline $6.25(2)^{d}$ & $53.04 \pm 3$ & $74.8 \pm 9$ & $5.51 \pm 2$ & $49.13 \pm 1$ & $8.95 \pm 3$ & $46.7 \pm 3$ \\
\hline 7.5 & $59.64 \pm 2$ & $89.1 \pm 6$ & $12.44 \pm 1$ & $47.53 \pm 2$ & $10.84 \pm 2$ & $48.8 \pm 1$ \\
\hline 10 & $55.14 \pm 2$ & $74.2 \pm 4$ & $9.52 \pm 2$ & $45.52 \pm 2$ & $8.84 \pm 1$ & $46.3 \pm 1$ \\
\hline 12.5 & $48.01 \pm 2$ & $60.4 \pm 2$ & $8.95 \pm 1$ & $41.22 \pm 4$ & $8.24 \pm 1$ & $41.6 \pm 4$ \\
\hline
\end{tabular}

a Temperature $22^{\circ} \mathrm{C}$, salinity $36.52 \%$. ' ${ }^{b}$ Theoretical values for $K_{0}$ and $K_{\mathrm{x}}$ using eq 7 and eq 8 , respectively. 'Values corresponding to lindane sorption-desorption at 45 (1) and $5{ }^{\circ} \mathrm{C}(2)$. ${ }^{d}$ Values corresponding to lindane sorption-desorption at salinities of 15 (1) and $25 \%$ (2).

several (approximately five) washes, the solids were airdried and used in the batch experiments. Preliminary studies with different fractions ranging from $250 \mu \mathrm{m}$ to less than $88 \mu \mathrm{m}$ gave sorption results similar to those of the bulk, which was used in all the following studies. Gran Canaria (Canary Islands) seawater (salinity, $S=36.52 \%$ ) was extracted with hexane to remove dissolved organic compounds and filtered $(0.45-\mu \mathrm{m}$ membrane filter) prior to use. A stock solution of lindane (Supelco, Inc. Bellefonte) in pesticide grade hexane $(0.75 \mathrm{ppm})$ was prepared.

Sorption experiments (in triplicate) were conducted by preequilibrating the lindane (ranging from 1 to $450 \mu \mathrm{g} / \mathrm{L}$ ) with $40 \mathrm{~mL}$ of seawater in $50-\mathrm{mL}$ glass-stoppered centrifuge glass tubes. After 24 -h preequilibration periods, the mass of chitin was added (from 2.5 to $12.5 \mathrm{~g} / \mathrm{L}$ ) and the glass tubes were shaken in the controlled temperature bath for 24-h sorption and 24-h desorption time periods. Subsequently, the samples were centrifuged at $6000 \mathrm{rpm}$ for $15 \mathrm{~min}$, after which $25 \mathrm{~mL}$ of supernatant was withdrawn and extracted with $10 \mathrm{~mL}$ of hexane. The content of lindane in these extracts was then determined by gas chromatography with an ECD detector. The pesticide mass was derived from triplicate measurements by comparing their peak area responses to a standard curve prepared with standard solutions of the pesticide in the same experimental conditions. The coefficients of variation of these triplicate measurements were $\sim 1 \%$. In such conditions, any loss of lindane from aqueous solution (volatilization or sorption to the walls of the centrifuge tube) is compensated by using the standard curve. Thus, it was assumed that the difference between the pesticides mass added and the pesticide mass in the liquid phase at the end was the result of chitin sorption.

For the desorption, the following procedure was repeated several times: the supernatant was replaced with contaminant-free seawater, shaken again for 24 -h periods, and recentrifuged, and the new supernatant was analyzed. Where it was necessary, the extract was concentrated to $1 \mathrm{~mL}$ to obtain detectable peaks.

\section{Results and Discussion}

Partitioning of lindane $(187.7 \mu \mathrm{g} / \mathrm{L})$ to chitin $(6.25 \mathrm{~g} / \mathrm{L})$ as a function of the equilibrium time showed that the process occurs quite rapidly. In $\sim 3 \mathrm{~h}$, the sorption process was complete and an increase in sorption time to $100 \mathrm{did}$ not show notable effects. For the desorption rate experiments, the process was essentially complete in a few minutes. To ensure equilibrium conditions, 24-h equilibrium periods, both for sorption and desorption experiments, were used in all the studies. For these time scales, a metastable equilibrium can be assumed and equilibrium

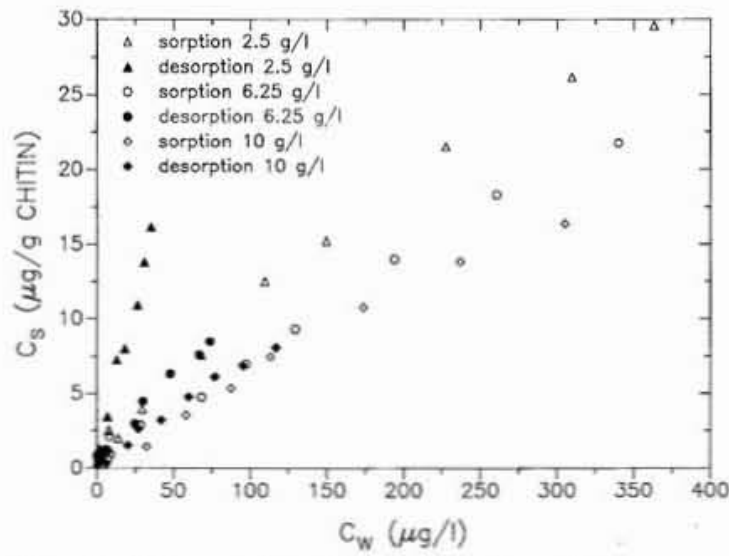

Figure 1. Lindane sorption and desorption isotherms for three representative chitin concentrations. Sorption and desorption equilibration time, $24 \mathrm{~h}$. Data are averages of three replicates.

models can be used properly. Sorption and desorption isotherms representative of those obtained for different amounts of chitin (ranging from 2.5 to $12.5 \mathrm{~g} / \mathrm{L}$ ) are presented in Figure 1. The isotherms have been interpreted in terms of the linear relationship (initial lindane concentration below $450 \mu \mathrm{g} / \mathrm{L}$ )

$$
C_{\mathrm{s}}=K C_{\mathrm{w}}
$$

where $K$ is an equilibrium constant, $C_{\mathrm{s}}$ is the sorbed lindane concentration $(\mu \mathrm{g} / \mathrm{g})$ on chitin, and $C_{\mathrm{w}}$ is the aqueous lindane concentration $(\mu \mathrm{g} / \mathrm{L})$. For the sorption isotherms, $C_{\mathrm{a}, \mathrm{a}}$ is related to $C_{\mathrm{w}, \mathrm{a}}$ and the partition coefficient for sorption is $K_{\mathrm{a}}$. Similarly, if the desorption points fall in a straight line, a desorption isotherm can be defined $C_{\mathrm{s}, \mathrm{d}}$ $=K_{\mathrm{p}, \mathrm{d}} C_{\mathrm{wd}, \mathrm{d}}$, with a partition coefficient $K_{\mathrm{p}, \mathrm{d}}(8)$. This curve is called a single desorption isotherm. In Table I, there is a list of the sorption and desorption partition coefficients for studies with different amounts of chitin. The differences in values of $K$ for sorption and desorption appear to indicate that the sorption process is not readily reversible and that it is dependent on the chitin concentration. Many examples of these isotherm pairs, where the desorption partition coefficients $K_{\mathrm{p}, \mathrm{d}}$ are greater than the sorption partition coefficient $K_{\mathrm{a}}$, are to be found in the existing literature $(8,25-27)$. In Figure 2, the results for the sorption and consecutive desorption data $(4 \mathrm{~g} / \mathrm{L})$ in four lindane concentrations (three replicates) are shown. For each lindane concentration, these points can also be described by a linear isotherm, which may be classified as the consecutive desorption isotherm, in accordance with previous studies $(7-11,28-30)$. 


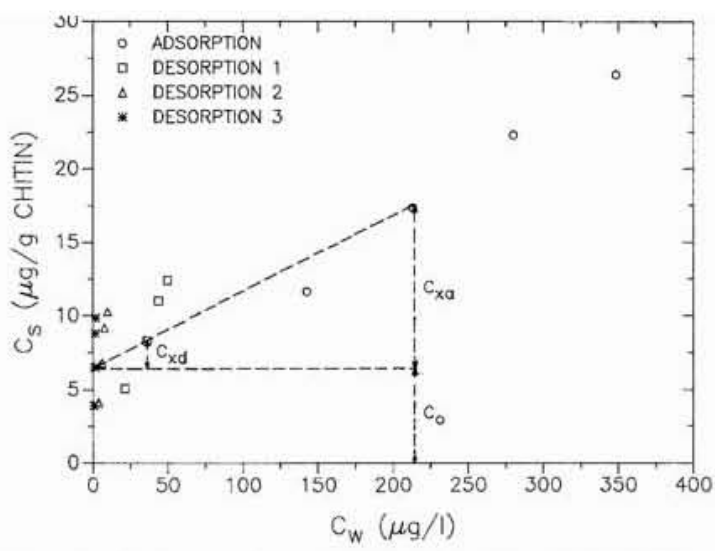

Figure 2. Lindane sorption and consecutive desorption data $(4 \mathrm{~g} / \mathrm{L}$ chitin concentration). Single and consecutive desorption data conform to the linear isotherms.

The results of the experimental consecutive desorption studies for all the concentrations indicate that there exists a significant component of the sorbed lindane which is extremely difficult to desorb. It is clear that for lindane (see Figure 2 and for other sorbates-adsorbents refs 8 and 26-30) the particulate lindane concentrations for the initial few desorptions are significantly above the sorption isotherms. However, at high chitin concentration (above 10 $\mathrm{mg} / \mathrm{L}$ ), both the sorption and the desorption isotherms are almost the same (see, for example, Figure 1 and Table I).

It has been suggested by Gschwend and Wu (13) and Baker et al. (14) that these two phenomena, the nonsingularity of the sorption-desorption process and the adsorbent concentration effect, may be attributed to nonsettling microparticles and dissolved macromolecules. Experiments carried out using washed chitin (in accordance with ref 13) and as described in the Experimental Section), after eliminating dissolved macromolecules and most of the nonsettling particles by determining the turbidity (absorbance at $500 \mathrm{~nm}$ in 10-cm cell in the spectrophotometer (13)) of the supernatant, did not produce significant effects on the lindane sorption and desorption isotherms of chitin. However, we found that when other factors such as temperature and salinity were changed, the sorption-desorption hysteresis observed was dramatically reduced (Table I).

The nonreversible behavior of sorption and desorption of lindane on chitin and the concentration effect have been studied by defining reversible and resistant components for the adsorbed state in the same way as Di Toro and Horzempa (8). The sorption and desorption isotherms of lindane to chitin are assumed to be linear in the concentration range studied ( 1 to $450 \mu \mathrm{g} / \mathrm{L}$ lindane). As can be seen in Figure 2, the assumption that the continued desorption cycles follow a straight line is well supported. The resistant component concentration, $C_{0}$, is defined as the extrapolated intersection of the consecutive isotherm and the ordinate, which resists desorption initially and may be completely nonexchangeable. The reversible component at sorption, $C_{x, a}$, and at the first desorption, $C_{x, d}$, corresponding to aqueous concentration $C_{\mathrm{w}, \mathrm{a}}$ and $C_{\mathrm{wd}}$, respectively, is defined as the difference between the sorbed lindane concentration at the sorption and desorption points and the extrapolated resistant concentration (Figure 2):

$$
\begin{aligned}
& C_{\mathrm{x}, \mathrm{a}}=C_{\mathrm{s}, \mathrm{a}}-C_{\mathrm{o}} \\
& C_{\mathrm{x}, \mathrm{d}}=C_{\mathrm{s}, \mathrm{d}}-C_{\mathrm{o}}
\end{aligned}
$$

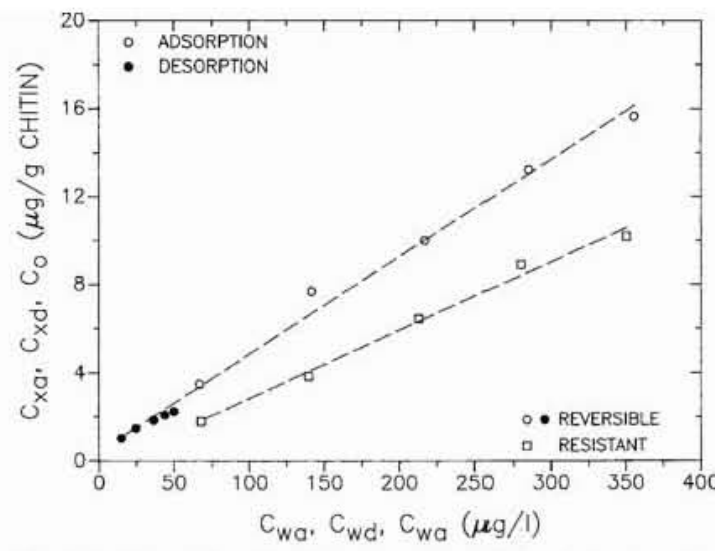

Flgure 3. Reversible and resistant component linear isotherms. The reversible sorption (eq 2) and reversible desorption (eq 3) points are described by the same single and linear isotherm, eq 5 . The resistant component was analyzed by using eq 4 , and the linear isotherm, eq 6 was from data in Figure 2.

From the geometry of the linear isotherms, the reversible and resistant components can be computed for each pair of sorption $\left(C_{\mathrm{s}, \mathrm{a}}, C_{\mathrm{w}, \mathrm{a}}\right)$ and single desorption $\left(C_{\mathrm{s}, \mathrm{d}}, C_{\mathrm{w}, \mathrm{d}}\right)$ values. The equation for the resistant component is (8)

$$
C_{\mathrm{o}}=\left(C_{\mathrm{s}, \mathrm{d}}-\beta C_{\mathrm{s}, \mathrm{a}}\right) /(1-\beta)
$$

where $\beta=C_{\mathrm{w}, \mathrm{d}} / C_{\mathrm{w}, \mathrm{a}}$. The reversible components can be computed by the difference, eqs 2 and 3 .

The validity of this analysis depends upon the observation that all the reversible component data conform to a single isotherm, either at sorption or at desorption equilibrium concentrations. Figure 3 shows the sorptiondesorption data plotted in Figure 2 analyzed using eqs 2-4. They demonstrate that indeed all the reversible sorption and desorption points may be described by a single and linear isotherm:

$$
C_{\mathrm{x}}=K_{\mathrm{x}} C_{\mathrm{w}}
$$

Figure 3 illustrates that for the resistant component a linear function of equilibrium sorption aqueous concentration, $C_{\mathrm{w}, \mathrm{a}}$, is obtained, leading to an isotherm for the resistant component

$$
C_{\mathrm{o}}=K_{\mathrm{o}} C_{\mathrm{w}, \mathrm{a}}
$$

The isotherm parameters for these data and for all the chitin concentrations studied are shown in Table I.

The $K_{\mathrm{o}}$ and $K_{\mathrm{x}}$ values determined by using the theoretical eqs 7 and 8 (Table I) derived by Di Toro et al. (8)

$$
\begin{gathered}
K_{\mathrm{o}}=\left[m K_{\mathrm{a}}\left(K_{\mathrm{p}, \mathrm{d}}-K_{\mathrm{a}}\right)\right] /\left[1+m\left(K_{\mathrm{p}, \mathrm{d}}-K_{\mathrm{a}}\right)\right] \\
K_{\mathrm{x}}=K_{\mathrm{a}} /\left[1+m\left(K_{\mathrm{p}, \mathrm{d}}-K_{\mathrm{a}}\right)\right]
\end{gathered}
$$

show that there exists a great deal of accordance between the experimental and theoretical data which support the theoretical model.

A second focus of the experiments carried out with lindane was the effect of the concentration of chitin on the partition coefficients. The variations of the sorption, $K_{a}$ and desorption, $K_{\mathrm{p}, \mathrm{d}}$, partition coefficients as a function of adsorbent concentrations are shown in Figure 4. The analysis of Figure 4 shows that both sorption and single desorption partition coefficients decrease as adsorbent concentration increases, as is the case with the nonreversibility of sorption-desorption. These data have been interpreted in terms of the reversible and resistant com- 


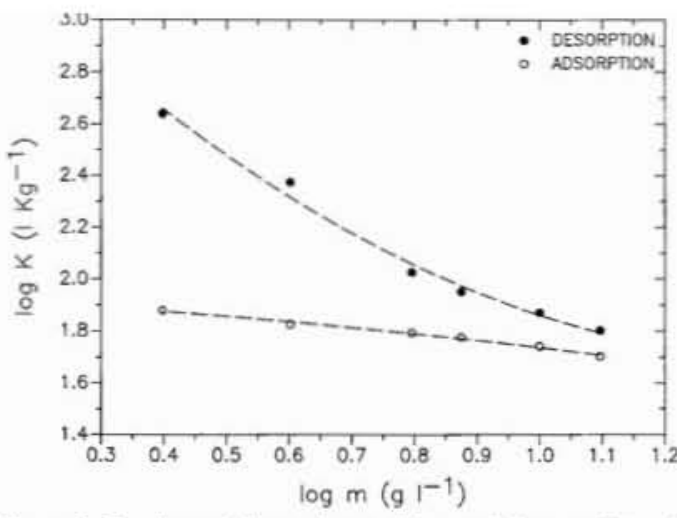

Flgure 4. Sorption and desorption partition coefficient variation with sorbent concentration. Curves are eq 12 for sorption and eq 13 for desorption partition coefficients.

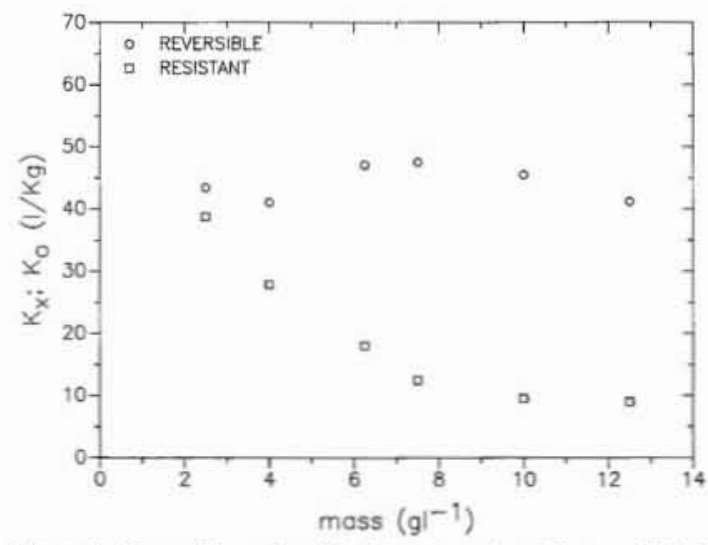

Flgure 5. Reversible and resistant component partition coefficient variation with adsorbent concentration. $K_{\mathrm{x}}$ is found to be constant with respect to chitin concentration.

ponent model. It is found (Figure 5) for the chitin that the reversible component partition coefficient, $K_{\mathrm{z}}$, is independent of the adsorbent concentration whereas the resistant component partition coefficient, $K_{\mathrm{o}}$, is dependent on the chitin concentration, and it is inversely correlated to the adsorbent concentration (Figure 6). This inverse relationship between $K_{0}$ and $m$, i.e.

$$
K_{\mathrm{o}}=\nu_{\mathrm{o}} m^{-1}
$$

defines $\nu_{0}$, a dimensionless distribution coefficient for the resistant component. This provides a consistent description of the reversible and resistant component partition coefficient, which requires two parameters only: $K_{\mathrm{x}}$ and the resistant component distribution coefficient $v_{0}$. In our case, $K_{\mathrm{x}}=45 \pm 4(\mathrm{~L} / \mathrm{kg})$ and $\nu_{0}=0.0978 \pm 0.007$. With these two known values, it is possible to relate the variations of sorption, $K_{w}$, and desorption, $K_{\mathrm{p}, \mathrm{d}}$, partition coefficients as functions of the adsorbent concentration. For $K_{x}$, it follows (3) that

$$
K_{\mathrm{x}}=\left[K_{\mathrm{a}}-K_{\mathrm{p}, \mathrm{d}}\left(C_{\mathrm{w}, \mathrm{d}} / C_{\mathrm{w}, \mathrm{a}}\right)\right] /\left[1-\left(C_{\mathrm{w}, \mathrm{d}} / C_{\mathrm{w}, \mathrm{a}}\right)\right]
$$

The relationship between $C_{w}$ and $C_{\mathrm{wd}}$ follows from the mass balance relationship $m C_{\mathrm{s}, \mathrm{s}}=C_{\mathrm{T}, \mathrm{d}}$; that is, the total lindane concentration in the desorption experiments is the same as the sorbed amount in the sorption experiments, and by using the fact that $C_{\mathrm{T}, \mathrm{d}}=C_{\mathrm{w}, \mathrm{d}}+m C_{\mathrm{s}, \mathrm{d}}$, so that

$$
C_{\mathrm{wd}} / C_{\mathrm{w}, \mathrm{a}}=m K_{\mathrm{a}} /\left(1+m K_{\mathrm{p}, \mathrm{d}}\right)
$$

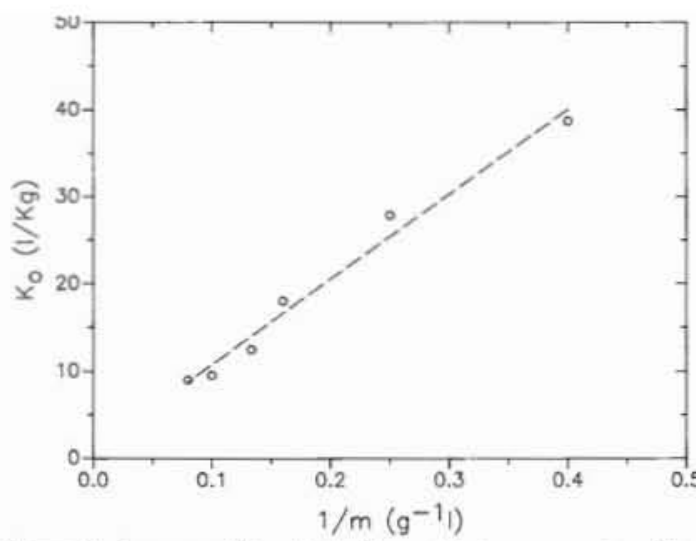

Flgure 6. Inverse relationship for the resistant component partition coefficient with chitin concentration. The slope, $0.0978 \pm 0.007$ corresponds to the dimensionless distribution coefficient for the resistant component, $\nu_{0}$.

By using these equations, determining $K_{\mathrm{a}}$ and $K_{\mathrm{p}, \mathrm{d}}$ in terms of $K_{\mathrm{o}}$ and $K_{\mathrm{x}}$, and using eq 9 for $K_{\mathrm{o}}$, then

$$
\begin{gathered}
K_{\mathrm{a}}=K_{\mathrm{x}}+\nu_{\mathrm{o}} / m \\
K_{\mathrm{d}}=K_{\mathrm{x}}+\left(\frac{v_{\mathrm{o}}}{m}\right)\left(1+1 / K_{\mathrm{x}}\right)
\end{gathered}
$$

This behavior of the partition coefficient for the reversible and resistant components implies that if the ratio of $K_{\mathrm{p}, \mathrm{d}}$ and $K_{\mathrm{a}}$ is used as the measure of nonreversibility, then

$$
\frac{K_{\mathrm{p}, \mathrm{d}}}{K_{\mathrm{a}}}=\frac{K_{\mathrm{x}}+\left(\frac{v_{\mathrm{o}}}{m}\right)\left[1+1 /\left(m K_{\mathrm{x}}\right)\right]}{K_{\mathrm{x}}+\nu_{\mathrm{o}} / m}
$$

So that

$$
K_{\mathrm{p}, \mathrm{d}} / K_{\mathrm{a}} \rightarrow 1 \quad \text { as } \nu_{\mathrm{o}} / m \rightarrow 0
$$

and

$$
K_{\mathrm{p}, \mathrm{d}} / K_{\mathrm{a}} \rightarrow 1+1 / m K_{\mathrm{x}} \quad \text { as } \nu_{\mathrm{o}} / m \rightarrow \infty
$$

These results model the observed increase of nonreversible behavior as the adsorbent mass decreases and they also give the quantitative explanation for the almost reversible behavior of the chitin at high concentrations.

If the description of the nonreversibility index is the difference between $K_{\mathrm{p}, \mathrm{d}}$ and $K_{\mathrm{a}}$ then eqs 12 and 13 predict that

$$
K_{\mathrm{p}, \mathrm{d}}-K_{\mathrm{a}}=\nu_{\mathrm{o}} / m^{2} K_{\mathrm{x}}
$$

which is dependent on the chitin concentration and where the slope $\nu_{\mathrm{o}} / K_{\mathrm{x}}=2.2057 \pm 0.0645$.

The partition coefficient behavior both for the sorption and desorption $K_{\mathrm{a}}$ and $K_{\mathrm{p}}$ and for the reversible and resistant components observed for the lindane sorption and desorption to chitin is found to be opposite to that found by Di Toro et al. (3) for the adsorption of HCB to Saginaw Bay sediments. They found, first, that nonreversibility increases as sediment concentration increases and, second, that while the resistant component partition coefficient, $K_{\text {o }}$, was independent of adsorbent concentration, the reversible component partition coefficient, $K_{\mathbf{y}}$, was inversely proportional to adsorbent concentration, which defines \& dimensionless distribution coefficient for the reversible component. The Di Toro et al. (3) experiments were 
carried out by using different sediments and natural particles with very low organic carbon contents (ranging from 0 to $5.4 \%$ organic matter versus $40 \%$ organic carbon for chitin) in natural and distilled waters. Seawater was used in our studies. The organic compound selected was HBC, a highly hydrophobic compound with a $\log K_{\text {ow }}=$ $7.36 \pm 0.43$. There are many differences between the physical and chemical properties in the phases involved in both studies. Thus, different sorption mechanisms can influence the final result. As we show later, lindane sorption in water of very low salinity behaves quite reversibly and the use of the mathematical model described by Di Toro et al. (12) is a very useful way of describing the sorption-desorption process in seawater when hysteresis is observed and kinetics do not account for the experimental data.

To gain insight into these phenomena, different studies at three temperatures $\left(5,22\right.$, and $\left.45^{\circ} \mathrm{C}\right)$ and three salinities $(15,25$, and $36 \%$ ) were carried out.

When the sorption and desorption experiments are carried out at three different temperatures, again a general trend is observed. Using the data shown in Table I, it is concluded, first, that an increase in temperature decrease the lindane sorption and, second, that at high temperatures the process becomes reversible $\left(K_{\mathrm{p}, \mathrm{d}}=K_{\mathrm{a}}\right)$. When the two-component model is applied to this system (Table I) we observe, first, that the reversible component is not affected by the temperature and, second, that the resistant component decreases as temperature increases and it alone is affected by the temperature. At high temperatures, the diffusion velocity of the lindane molecules increases; the attractive forces between the solute and the chitin surface may decrease (12), resulting in greater desorption.

Other experiments were conducted to study the effect of salinity on the sorption of lindane. Using the data shown in Table I, it is observed that an increase in salinity from $S=15 \%$ to $S=36 \%$ increases the sorption and desorption partition coefficients and increases the degree of nonreversibility. Wildish et al. (32) reported increased hysteresis of desorption isotherms when PCBs adsorbed in distilled water were then desorbed in water of increasing salinity. Again, if the reversible-resistant model is applied, the general trend of a constant reversible component and a resistant component which decreases as salinity decreases is observed. A linear relation between $K_{\mathrm{o}}$ and $\sqrt{ } S$ is found with a slope of $7.048( \pm 0.02)$. It means ionic strength is only affecting the process accounting for the existence of a resistant component. When sorption-desorption reactions are carried out in seawater, its high ionic concentration may modify both the heterogeneous surface properties of the solid particles and the sorbate properties. It is well assumed that in the consideration of hydrophobic substances, gross electrostatic interactions are usually negligible, but attraction potentials can be developed between polar molecules and ionic or heteropolar solids. The strength of this attraction is dependent upon the strength and characteristics of the surface electric field and the magnitude of the dipole and quadrupole moments of the solute molecules. A light degree of polarity can radically alter the stability of molecules in solution, thereby exerting considerable control over sorption. The possible effect of ionic strength and major ion chemistry on the organic phase is uncertain. If increased ionic strength acts to reduce the water content of the organic phase, it might enhance the compatibility of larger HOC with the organic phase, resulting in greater partitioning. On the other hand, the ionic strength influences sorption by affecting the surface charge and the double-layer properties of the hy- drated particles. At high salinities, the increase of lindane sorption may be the result of the neutralization and the development of positively charged sites on the chitin particle edges resulting from cation adsorption from seawater (at $\mathrm{pH}$ values lower than 4 , partition coefficients 3 times greater than those for the $\mathrm{pH}$ of seawater were observed, and chitin has been found to be a good adsorbent for metal ions (20-23)). Taking into account these effects, we can assume the sorbing capacity of chitin in seawater observed in the present study may be the result of chitin-lindane interactions of a different nature due to the heterogeneity of the chitin molecule. A hydrophobic together with an electrostatic interaction between the chitin surface groups (hydroxyl, acetylamino groups, and adsorbed cations) and the lightly polar lindane can account for the observed effects. Using the results obtained by the two-component model, the first interaction can be described by a reversible component partition coefficient, as in many other studies of HOC partitioning in soil and sediment in natural and distilled water. The second interaction will be described by the nonreversible partition coefficient. This coefficient is affected by both the salinity and chitin concentration. A decrease in salinity will affect the chitin surface properties, decreasing the concentration of adsorbed cations, and, as a consequence, most of the electrostatic nature interactions will disappear. Thus, the resistant component partition coefficient $K_{0}$ will tend to lower values (Table I). In distilled water and natural waters, where most of the research work has been carried out, the process behaves reversibly and is not affected by solid concentration (studies carried out at different salinities with $m=12.5 \mathrm{~g} / \mathrm{L}$ showed similar results).

When the effect of chitin concentration is taken into account, it is observed that only the resistant component $K_{\mathrm{o}}$ is affected. It implies that by increasing solid concentration the particles tend to "communicate" to a greater extent, thus blocking potential surface sorption sites of an electrostatic nature as the result of increased aggregation (33). At high chitin concentration, chitin-lindane interactions of a hydrophobic nature contribute most to the partitioning reaction and the process becomes reversible. However, the mechanism that accounts for this behavior is not clear yet, and further investigations for adsorbates and/or adsorbents in seawater are required.

\section{Conclusion}

It appears that the description of lindane sorption-desorption to chitin in seawater in terms of reversible and resistant components is certainly successful and can model most of the observed effects. The chitin concentration effects, the nonsingularity of sorption and desorption processes, and the effects of temperature and salinity show a general trend: the reversible component partition coefficient is fairly constant while the resistant component partition coefficient is inversely related to the chitin concentration. At a fixed chitin concentration, the resistant component is inversely proportional to temperature and is directly related to the salinity. At high temperatures and low salinities, the process becomes reversible and the resistant component partition coefficient becomes zero. These effects can clarify the mechanisms responsible for hysteric isotherms and adsorbent concentration effects. It has been pointed out that sorption of lindane in seawater may take place both by hydrophobic interaction described by a reversible partition coefficient and by electrostatic interaction described by a resistant partition coefficient which is affected by salinity and the temperature of the solvent. However, the description of the different effects is not yet complete and additional experimental and the- 
oretical investigations are required.

Registry No. Lindane, 58-89-9; chitin, 1398-61-4.

\section{Literature Cited}

(1) Grover, R.; Hance, R. J. J. Soil Sci. 1970, 109, 136-138.

(2) O'Connor, D. J.; Connolly, J. P. Water Res. 1980, 14, 1517-1523.

(3) Di Toro, D. M.; Horzempa, L. M.; Casey, M. M.; Richardson, W. J. Great Lakes Res. 1982, 8, 336-349.

(4) Voice, T. C.; Rice, C. P.; Weber, W. J., Jr. Environ. Sci. Technol. 1983, 17, 513-518.

(5) Bowman, B. T.; Sans, W. W. J. Environ. Qual. 1985, 14, 265-269.

(6) Coates, J. T.; Elzerman, A. W. J. Contam. Hydrol. 1986, $1,191-210$.

(7) Rao, P. S. C.; Davidson, J. In Environmental Impact of Nonpoint Source Pollution; Overcash, M. R., Davidson, J. M., Eds.; Ann Arbor Science: Ann Arbor, MI, 1980; pp 23-67.

(8) Di Toro, D. M.; Horzempa, L. M. Environ. Sci. Technol. $1982,16,594-602$.

(9) Swanson, R. A.; Dutt, G. R. Soil Sci. Soc. Am. Proc. 1973, $37,872-876$

(10) Peck, D. E.; Corwin, D. L.; Farmer, W. J. J. Environ. Qual. $1982,16,735-740$.

(11) Wahid, P. A.; Sethunathan, N. J. Agric. Food Chem. 1979, $27,1050-1053$

(12) Di Toro, D. M.; Horzempa, L. M.; Casey, M. C. Adsorption and Desorption of Hexachlorobiphenyl. Final report, Environmental Engineering and Science Program, Manhattan College, Bronx, NY, 1982.

(13) Gschwend, P. M.; Wu, S.-C. Environ. Sci. Technol. 1985, $19,90-96$.

(14) Baker, J. E.; Capel, P. D.; Eisenreich, S. J. Environ. Sci. Technol. 1986, 20, 1136-1143.

(15) Jaffé, P. R. J. Environ. Sci. Health 1986, A21, 55-69.

(16) Witkowski, P. J.; Jaffe, P. R.; Ferrara, R. A. J. Contam. Hydrol. 1988, 2, 249-269.
(17) Chin, Y.-P.; Weber, W. J., Jr.; Eadie, B. J. Environ. Sci. Technol. 1990, 24, 837-842.

(18) Caron, G.; Suffet, I. H.; Belton, T. Chemosphere 1985, 14, 993-1000.

(19) Smith, E. H. Water Res. 1991, 25, 125-134.

(20) González-Dávila, M.; Santana-Casiano, J. M.; Millero, F. J. J. Colloid Interface Sci. 1990, 137, 102-110.

(21) González-Dávila, M.; Millero, F. J. Geochim. Cosmochim. Acta 1990, 54, 761-768.

(22) Muzzarelli, R. A. A. Chitin; Pergamon: London, 1977.

(23) Muzzarelli, R. A. A. Natural Chelating Polymers; Pergamon: London, 1973; Chapters 3, 5.

(24) Allison, L. B. In Methods of Soil Analysis; Black, C. A., Ed.; American Society of Agronomy: Madison, WI, 1965; Vol. II, Chapter 90.

(25) Felsot, A.; Dahm, P. A. J. Agric. Food Chem. 1979, 27, 557-563.

(26) Pierce, R. H., Jr.; Olney, C. E.; Felbeck, G. T., Jr. Geochim. Cosmochim. Acta 1980, 24, 20-26.

(27) Koskinen, W. C.; O'Connor, G. A.; Cheng, H. H. Soil Sci. Soc. Am. J. 1979, 43, 871-874.

(28) van Genuchten, M. Th.; Davidson, J. M.; Wierenga, P. J. Soil Sci. Soc. Am. J. 1974, 38, 29-35.

(29) van Genuchten, M. Th.; Wieranga, P. J.; O'Connor, G. A. Soil Sci. Soc. Am. J. 1977, 41, 278-285.

(30) Wahid, P. A.; Sethunathan, N. J. Agric. Food Chem. 1978, $26,101-105$.

(31) Di Toro, D. M.; Horzempa, L. M. Physical Behavior of PCB's in The Great Lakes; Mackay, D., Peterson, S., Eisenrich, S. J., Simmons, M. D., Eds.; Ann Arbor Science: Ann Arbor, MI, 1983; pp 89-113.

(32) Wildish, D. J.; Metcalfe, C. D.; Akagi, H. M. Mcleese, D. W. Bull. Environ. Contam. Toxicol. 1980, 24, 20-26.

(33) Brownawell, B. J. Ph.D. Dissertation, Woods Hole Oceanographic Institute/MIT, 1986.

Received for review January 15, 1991. Revised manuscript received June 13, 1991. Accepted July 29, 1991. 